\title{
Programas socioeducativos y regulación del trabajo docente en el nivel secundario'
}

\author{
Analía Inés Meo"
}

Diana Milstein"II

I- Los autores agradecen la colaboración de adultos y jóvenes de la escuela de este estudio. II- Instituto de Investigaciones Gino Germani, Universidad de Buenos Aires, CONICET, Ciudad Autónoma de Buenos Aires, Argentina. Contacto: analiameo@conicet.gov.ar

III- Facultad de Ciencias de la Educación, Universidad Nacional del Comahue, Provincia de Rio Negro y Centro de Investigaciones Sociales, CONICET, Instituto de Desarrollo Económico y Social, Ciudad Autónoma de Buenos Aires. Argentina.

Contacto: diana_mils@yahoo.com.ar

\section{Resumen}

Durante las últimas tres décadas, los gobiernos nacionales y provinciales han implementado diferentes tipos de políticas para modificar aspectos centrales de la enseñanza secundaria estatal en Argentina. En este trabajo examinaremos, a partir del análisis etnográfico de un programa socioeducativo, cómo las políticas educativas, instaladas en los 90 y profundizadas en los 2000 -que sostienen una retórica tendiente a producir más autonomía escolar- están redefiniendo aspectos centrales del trabajo docente. Analizaremos el Programa de Fortalecimiento Institucional de la Escuela Media de la ciudad de Buenos Aires y los efectos prácticos que produjo esta modalidad de intervención estatal, respecto del gobierno escolar, de las relaciones de poder en la escuela, y de las condiciones de trabajo de docentes de una escuela secundaria que trabaja con jóvenes de familias vulnerables en el sur de la ciudad. Para ello, en primer lugar, situaremos nuestra investigación en el campo de producción de conocimiento local. Luego, presentaremos la historia de las Escuelas Municipales de Educación Media y mostraremos cómo su creación representó una transformación del gobierno escolar en el nivel medio en la Ciudad. Seguidamente, focalizaremos nuestra atención en el programa Fortalecimiento como modalidad de regulación institucional estatal y, a continuación, nos detendremos en la descripción del proyecto escolar El Puente. Este recorrido nos permitirá evidenciar cambios significativos en el gobierno escolar, las relaciones entre directores y docentes, así como la precarización e intensificación de su trabajo. Realizamos nuestro trabajo de campo entre noviembre de 2012 y diciembre de 2014.

\section{Palabras clave}

Programas socioeducativos - Trabajo docente - Nivel secundario. 


\section{Social-educational programs and the regulation of teachers' work in secondary education'}

Analía Inés Meo"

Diana Milstein"II

I- The authors are grateful for the collaboration of adults and young people from the school in this study.

II- Instituto de Investigaciones Gino Germani, Universidad de Buenos Aires, CONICET, Ciudad Autónoma de Buenos Aires, Argentina.

Contact: analiameo@conicet.gov.ar

III- Facultad de Ciencias de la Educación, Universidad Nacional del Comahue, Provincia de Rio Negro y Centro de Investigaciones Sociales, CONICET, Instituto de Desarrollo Económico y Social, Ciudad Autónoma de Buenos Aires. Argentina. Contact: diana_mils@yahoo.com.ar

\begin{abstract}
Over the last three decades, the national and provincial governments have implemented different types of policies aiming to transform key aspects of state secondary schooling in Argentina. In this paper, based on an ethnographic study on a social-educational program, we examine how educational policies deployed in the 1990s have continued to expand in the 2000s - and how they are redefining significant features of teachers' work. We analyze the Programa de Fortalecimiento Institucional de la Escuela Media in the city of Buenos Aires and its practical effects in terms of school government, power relations in schools, and teachers' working conditions in a secondary school, whose students are young people from vulnerable families, in the south of the city. Firstly, we characterize the local field of knowledge production and locate our research within it. Secondly, we present the history of Escuelas Municipales de Educacion Media and argue that their creation in 1990 represented a transformation in the school government of secondary education. Thirdly, we unpack how Fortalecimiento operates. To do so, we scrutinize its key features and then we closely examine a proyecto escolar [school project] called El Puente. This examination allows us to identify key changes in the school government, in the relationships between the head teacher and teachers, as well as the intensification and worsening of teachers' working conditions. Fieldwork was carried out between November 2012 and December 2014.
\end{abstract}

\section{Keywords}

Social-educational programs - Teachers' work - Secondary education 


\section{Introducción'}

Durante las últimas tres décadas, los gobiernos nacionales y provinciales han implementado diferentes tipos de políticas para modificar aspectos centrales de la enseñanza secundaria estatal en Argentina. Estas políticas han estado en línea con tendencias educativas internacionales sustentadas en lo que denominaron proyectos de modernización ${ }^{2}$ del campo de la educación. Los propósitos declarados eran incorporar a jóvenes de amplios sectores sociales que históricamente quedaron restringidos en el acceso a la escuela media, actualizar los contenidos curriculares y a los profesores para que pudieran enseñarlos y corregir los efectos burocráticos negativos de la organización centralizada del sistema educativo. Así, las escuelas de nivel medio desde el inicio de la década del 90, con diferencias en distintas regiones de nuestro país, fueron objeto de reformas tendientes a producir la descentralización del sistema y a instalar formas nuevas de administración y financiamiento.

La primera oleada de reformas se produjo en la década de 1990 en el contexto de la reforma del Estado que incluyó la privatización de empresas estatales, la racionalización del gasto público, y la descentralización de organizaciones del Estado para dinamizar el mercado y la inserción de la Argentina en el primer mundo. Estas reformas produjeron hacia finales del siglo XX una verdadera catástrofe económica, política y social que para el sistema escolar implicó entre otros efectos, una parcial desarticulación, problemas agudos de financiamiento, y desorganización de las escuelas públicas. Entre 1999 y 2001 quedó opacada y maltrecha la ilusión modernizadora y comenzaron a

1- Una primera versión de este trabajo fue presentada en las V Jornadas de Etnografía y Procesos Educativos en Argentina, IDES, 24 y 25 de septiembre de 2015.

2- Las palabras y expresiones destacadas indican lenguaje utilizado en discursos periodísticos, políticos, académicos y por los mismos actores escolares.
[...] proliferar resoluciones, programas y proyectos especiales en los que se explicitaba la necesidad de formalizar/ regular redes de integración social y educativa, en las que intervinieran tanto las escuelas y los organismos gubernamentales de educación, minoridad y familia, como individuos y organizaciones de la sociedad civil. (GIOVINE, 2012, p. 29).

En el sistema educativo de la ciudad de Buenos Aires, esta tendencia a la emergencia de nuevas formas de regulación estatal destinadas a las escuelas medias, se materializó en diversos programas. Uno de ellos, denominado Programa de Fortalecimiento Institucional de la Escuela Media (de ahora en adelante nos referiremos a este como Fortalecimiento, que es la manera en que las autoridades y docentes se refieren a este programa) fue creado en el año 2000, se puso en marcha en el 2001 y continúa vigente. El propósito declarado era que, a través de proyectos seleccionados, financiados y regulados por Fortalecimiento, las escuelas estatales secundarias alcanzaran el objetivo de promover la retención escolar, para compensar lo que era considerado como un escenario con altos índices de deserción escolar y repetición.

Nuestra investigación se ha planteado, entre otros, el objetivo de describir y analizar la configuración de nuevas formas de trabajo de los docentes, los auxiliares y las autoridades en un proceso tendiente a reestructurar las escuelas estatales ${ }^{3}$. En función de esto desarrollamos trabajo de campo en la Escuela Municipal de Educación Media Susana Montes ${ }^{4}$, entre noviembre de 2012 y diciembre de 2014. Durante este período, además de desarrollar una intensa observación con participación ${ }^{5}$

3 - Este objetivo forma parte del Proyecto de Investigación PICT 20101356 Un nuevo lugar social para la escuela estatal. Entre la irrupción de la política y la emergencia de nuevas infancias y adolescencias. Investigadora responsable: Diana Milstein. Financiado por ANPCYT/FONCYT - Préstamos BID 2437.

4- Los nombres de instituciones y personas son seudónimos.

5- El trabajo de campo fue realizado por Analía Inés Meo, Linda Khodr y Verónica Solari Paz, acompañado y orientado por Diana Milstein. 
en la escuela ${ }^{6}$ y en el barrio, nos ocupamos de conocer la historia y el presente de los programas socioeducativos que operaban en la escuela, así como normativas, reglamentaciones $\mathrm{y}$ otros documentos.

En este artículo, nos proponemos identificar y analizar los cambios que introdujo la puesta en marcha de programas socioeducativos como Fortalecimiento que, en tanto modalidad original de intervención del Estado en el nivel secundario, ha producido cambios en el gobierno escolar, en las relaciones de poder en la escuela, en las condiciones de trabajo de los docentes, así como modificado algunos de los significados más extendidos de la autonomía escolar. Para ello, describimos y analizamos la generación e implementación del proyecto El Puente, el cual involucró cambios que, desde la perspectiva de los protagonistas y de otros actores escolares, impactaron la propia organización de la institución y el trabajo de los docentes.

\section{Los programas socioeducativos y el campo de producción de conocimiento local}

Desde la década de 1980, es posible rastrear variedad de estudios sobre procesos de reformas de los modos de regulación política centralizada que caracterizó a los sistemas educativos modernos, tanto en países centrales como en periféricos. En numerosos países industrializados occidentales, el estudio del gobierno escolar se ha centrado en fenómenos tales como la instauración de mecanismos de cuasi mercado y en la emergencia de actores del mundo de los negocios y de la sociedad civil en la organización y definición de aspectos centrales de lo que se enseña, cómo y para qué se lo hace (BALL; YOUDELL, 2007; BARROSO, 2003; DUPRIEZ; MAROY, 2003; MAROY, 2004; WHITTY; POWER, 2000). En Argentina, también existen estudios que han indagado los procesos

6- En el año 2014, la escuela tenía alrededor de 400 alumnos distribuidos en 13 clases. de transformación del gobierno escolar y de la reorganización del llamado Estado docente -caracterizado por su fuerte capacidad de regular a partir de marcos normativos y de principios jerárquicos producidos por el gobierno central (CARNOY; MOURA CASTRO, 1996; FELDFEBER, 2003). En general, estos estudios han mostrado distintos ritmos y características en los procesos de descentralización y algunos han mapeado distintos efectos (organizacionales, laborales y sociales) de su implementación. Sin embargo, no hemos encontrado estudios locales que hayan estudiado cómo los programas socioeducativos han alterado la forma del gobierno escolar en la vida cotidiana de las instituciones; y cómo se imbrican, alteran, tensionan o son resistidos en las escuelas.

Durante la primera década de los 2000, como parte de las numerosas transformaciones sociales, económicas y políticas, en el campo educativo se desplegaron políticas a nivel nacional de nuevo signo que se encarnaron en leyes (incluyendo una nueva ley de educación nacional), en la creación de dispositivos de intervención en las escuelas, y en el crecimiento del presupuesto educativo (DURO; PERAZZA, s/f; FELDFEBER; GLUZ, 2011; GOROSTIAGA, 2007). Sin embargo, en línea con las políticas establecidas en los 90 que buscaban promover la autonomía escolar, el Ministerio de Educación nacional y los provinciales - con diferentes ritmos y alcances- han creado y financiado programas, muchos de ellos incluidos dentro del rótulo socioeducativos, para incentivar, organizar y financiar proyectos escolares en las escuelas medias con el objetivo de implementar Proyectos Educativos Institucionales (TEDESCO; TENTI, 2004). El objetivo central declarado por parte de las autoridades era mejorar las tasas de retención y graduación escolar. Durante los últimos años los recursos destinados a estos programas se han incrementado (DABENIGNO et al., 2014; DURO; PERAZZA, s/f). Entre los programas más importantes, por su expansión y sostenimiento, mencionamos -a nivel nacionalel Plan de Mejora Institucional para la escuela 
secundaria y, en la Ciudad Autónoma de Buenos Aires (CABA), el Programa de Fortalecimiento Institucional de la Escuela Media (PFIEM) ${ }^{7}$.

Distintas investigaciones sociológicas $\mathrm{y}$ antropológicas han examinado programas socioeducativos (SINISI et al., 2003; MONTESINOS; SCHOO, 2014; TENTI FANFANI; FREDERIC; STEINBERG, 2009; DABENIGNO et al., 2014). Estos estudios, cualitativos en su mayoría, se han interrogado sobre cómo autoridades, docentes y alumnos interpretan la extensión de la obligatoriedad y la inclusión; el abandono y fracaso escolar; y la introducción de variadas políticas socio-educativas en las escuelas (tales como el programa de becas estudiantiles a nivel nacional y de la ciudad, el programa de retención de madres y padres, las tutorías, etc.). Orientaron sus análisis hacia los modos en que las agencias estatales educativas clasifican, nominan y producen distintos aspectos de la realidad escolar como problemas con soluciones. De ahí que estas investigaciones construyeron sus interrogantes a la luz de los objetivos de los programas y de las políticas, sus maneras de organizar, clasificar y conocer la realidad escolar y se inscriben en la tradición de las investigaciones orientadas a las politicas educativas (policy oriented research) (BALL; MAGUIRE; BRAUN, 2012). Desde esta perspectiva, las politicas educativas se consideran un campo, un dominio especializado o esfera claramente demarcado dentro de la realidad escolar. Así, estos abordajes tienden a perder de vista aspectos centrales de la materialidad, historia y relaciones de poder en las escuelas (BALL; MAGUIRE; BRAUN, 2012). En este sentido, no reconocen a las escuelas como sitios donde las políticas se tornan visibles en actuaciones, comportamientos y discursos de individuos y grupos que se expresan en la capilaridad de la vida social.

Nuestro estudio se interesa por comprender cómo las políticas educativas, instaladas en los 90 y profundizadas en los 2000

7- EI PFIEM es el antecedente directo de la creación y forma de trabajo del Plan de Mejora Institucional. están redefiniendo el gobierno y las relaciones de poder en las escuelas y transformando aspectos centrales del trabajo docente y cómo, paradójicamente, tienden a producir formas renovadas de intervención del Estado. Para ello, hemos incorporado como lenguaje nativo a los discursos emanados de las agencias estatales educativas, tal como son utilizados en la cotidianeidad de la vida de las escuelas y en la explicitación de las políticas a través de las normativas, las distintas formas de legislación y los fundamentos de los propios programas y proyectos. El término nativo remite al enfoque etnográfico en el que se encuadra nuestra investigación tanto en la modalidad del trabajo de campo como en los análisis y la escritura.

Hemos incorporado también en nuestros análisis, la noción de regulación para comprender la coexistencia de mecanismos de coordinación, orientación y control del sistema educativo producidos de arriba hacia abajo -a través de las decisiones y formas de control fijados por las autoridades políticas del sistema educativo- y de abajo hacia arriba -a través de las prácticas de los actores escolares (REYNAUD, 1987; MAROY; DUPRIEZ, 2000) ${ }^{8}$.

\section{Cambios en la forma de gobierno escolar}

\section{Las Escuelas Municipales de Educación Media, autonomía y regulación estatal}

En 1990, como resultado de la convergencia de diferentes orientaciones de política educativa, se crearon escuelas nuevas y diferentes en la ciudad de Buenos Aires. Se las llamó Escuelas Municipales de Enseñanza Media $(\text { EMEM })^{9}$ y fueron una novedad en un triple sentido. En primer lugar, fueron creadas por el gobierno municipal antes de la descentralización

8- El término regulación refiere a un proceso social de producción de reglas de juego (REYNAUD, 1997).

9- Luego de la descentralización del nivel secundario en la década de 1990, las Escuelas Municipales de Educación Media (EMEM) fueron rebautizadas como Escuelas de Educación Media (EEM). Con el objetivo de simplificar la lectura, hemos dejado la nominación original de las escuelas. 
de las escuelas nacionales estatales secundarias, y, al momento de su creación, dependían formalmente de la Dirección de Educación post-primaria. En segundo término, las EMEM fueron creadas en barrios donde había pocas escuelas secundarias con el objetivo explícito de brindar oportunidades educativas a jóvenes que, en general, sólo habían completado la escuela primaria o habían abandonado el nivel medio. En tercer lugar, la organización académica, social y pedagógica de las EMEM no estaba inscripta en marcos regulatorios propios. Aspectos tales como el plan de estudios, la asistencia de estudiantes, la evaluación y la disciplina no estaban reguladas por reglamentos, resoluciones o decretos específicos.

La creación de las EMEM fue, en parte, el resultado de la anticipación política del gobierno municipal al proceso de descentralización que se inició formalmente dos años después (MÁS ROCHA, 2006), cuando fueron transferidas administrativa y económicamente las escuelas secundarias del estado nacional. Esta transferencia fue experimentada por profesores, autoridades escolares, supervisores y sindicatos docentes como la retirada del Estado; como una imposición; como ataque a las condiciones de trabajo de los docentes, y a la calidad de la educación. En las EMEM, sin embargo, ocurrió una situación inédita: como parte del proceso de su creación no se establecieron marcos regulatorios propios del plan de estudios, ni de asistencia y comportamiento de los estudiantes, ni de nombramiento de docentes. Las EMEM adoptaban regulaciones pre-existentes de manera flexible y creativa con cierto margen de maniobra para definir la organización de la cotidianeidad escolar dentro y fuera del aula. Un supervisor narraba:

[...] cuando se armaron las escuelas (...) no había normativa (...) la otra vez se caracterizó a los directores de las EMEM históricas como "la gente que está acostumbrada a negar la normativa o a no responder a la normativa”. No, no teníamos normativa (...) no habia, estaba la normativa de nación y no dependíamos de nación (...). Municipalidad no tenía dirección de media, tenía post-primaria. (...) Nos reuníamos y decidíamos, después bueno, usamos el régimen de calificaciones de nación, no vamos a hacer uno nuevo. Se usó ese. Bueno, ¿qué hacemos con inasistencias? Amonestaciones nunca hubo, se trabajó desde los primeros años con consejo de convivencia, en 1990. (Entrevista a supervisor de escuelas secundarias, 26 de Mayo de $2011^{10}$ ).

Desde la perspectiva de las autoridades educativas del gobierno municipal y de las escuelas, las EMEM tendían a ampliar la cobertura de la educación secundaria porque incorporaron jóvenes pertenecientes a grupos sociales que históricamente no tuvieron acceso a este nivel educativo. Además, contaron con recursos presupuestarios específicos y tuvieron cierta vía libre para imaginar estrategias para obtener apoyos materiales y simbólicos de diferentes tipos de organizaciones -tales como equipos de investigación de la universidad y fondos asignados a programas destinados a captar estudiantes secundarios que estaban fuera del sistema.

Así, las EMEM en sus comienzos estaban en una relativa mejor posición que las escuelas municipalizadas después de haber sido transferidas desde el ámbito nacional. Eran más pequeñas y contaban con una proporción superior de profesores por estudiantes; tenían un asistente pedagógico y tutores, y contaban con el apoyo de figuras clave de la Secretaría de Educación municipal. Estos rasgos contribuyeron a que supervisores, directores, docentes y preceptores identificaran a estas escuelas como diferentes en varios aspectos. Lo diferente también se expresó, por ejemplo,

10- Esta entrevista fue realizada por Analía Meo como investigadora del proyecto de investigación UBACyT Las identidades laborales docentes en tiempos de fragmentación educativa. Un estudio de casos en la Ciudad de Buenos Aires (Código 20020110200110). Su directora fue Valeria Dabenigno. 
en las formas en que fueron seleccionados los primeros directores de las EMEM y gran parte del personal docente incorporado al comienzo, ya que no fue aplicada la normativa nacional que regulaba la contratación de autoridades. Los directores fueron elegidos por las autoridades educativas a través de designaciones personales, de acuerdo a consideraciones personalizadas de sus trayectorias y trabajo con jóvenes de grupos vulnerables, sus compromisos y sus credenciales políticas -adquiridas a través de su militancia o la participación política en el peronismo que gobernaba la ciudad de Buenos Aires (MÁS ROCHA, 2006).

En la escuela de nuestro estudio, Susana, la primera directora, tenía trato personal y cercano con autoridades de la sub-secretaría de Educación de la ciudad, había militado en una villa ${ }^{11}$ y participado activamente en el peronismo. Susana designó de manera personal al asistente pedagógico, a muchos docentes y preceptores, considerando sus experiencias previas con poblaciones similares a las de la EMEM (ya sea en escuelas primarias o secundarias, y/o en organizaciones políticas o sociales), su respeto hacia ellos y su interés en participar de la construcción de una escuela diferente. En relación con los primeros preceptores, Susana consideró que era fundamental que vivieran en la villa. Ellos tenían que conocer a los estudiantes, sus familias y condiciones de vida para forjar buenas relaciones con ellos y así poder dar respuesta a conflictos que emergieran entre los estudiantes (que, en general, los adultos asociaban con problemas que venian de la villa).

La elección de autoridades escolares y de algunos miembros del personal docente basada en sus cualidades personales y/o credenciales políticas o militantes fue una novedad dentro del sistema educativo porque las condiciones de trabajo y las formas de selección del personal docente y sus carreras en las escuelas estatales, estaban reguladas por el Estatuto del Docente -marco normativo que a nivel nacional existe

11- El término villa alude a villa miseria. desde 1958- con normas completamente ajenas a esta modalidad.

Diferentes directoras de las EMEM, docentes contratados al momento de la creación de esta escuela y preceptores, aseguraban que esta forma de designación personal era muy positiva porque había otorgado mayor margen de maniobra y había permitido la constitución de un grupo comprometido que aspiraba a responder creativamente a lo que ellos y Susana consideraban como necesidades de sus estudiantes. Esta forma de contratación promovió, desde el inicio, vínculos laborales basados en relaciones personales fundadas en la confianza, fuera esta generada en relaciones de amistad, familiaridad, actividad gremial o política.

\section{El Programa de Fortalecimiento como nuevo modo de regulación institucional}

A partir del año 2000, en la ciudad de Buenos Aires, se desplegaron programas educativos e iniciativas locales con el propósito declarado de garantizar el acceso, la permanencia y la graduación de los estudiantes secundarios. La creación del Programa de Fortalecimiento Institucional de la Escuela Media (PFIEM) en el 2000 se inscribió en ese abanico de políticas. Este programa fue acompañado por recursos materiales y humanos para las escuelas para alcanzar lo que se denominó la universalización del nivel medio. El propósito declarado por las autoridades fue brindar recursos adicionales a las escuelas para que llevaran adelante proyectos institucionales que se orientaran a la retención y apoyo de la enseñanza de los jóvenes. Este programa financió proyectos que fueron generados y llevados adelante por docentes en las escuelas y que buscaron alterar sus formas de trabajo y mejorar el involucramiento y rendimiento escolar de los estudiantes.

Recién en el año 2008, el gobierno aprobó el Manual o Pautas para que fueran aplicados en las escuelas de nivel medio dependientes de diversas direcciones ministeriales. En las distintas versiones del Manual el programa 
quedó definido como una estrategia para la construcción de las políticas públicas (BUENOS AIRES, 2009), cuyo objetivo central era "generar y consolidar aquellas condiciones institucionales favorables al trabajo de equipos docentes en proyectos que se orienten hacia la inclusión educativa de todos los estudiantes" (BUENOS AIRES, 2008, p. 7). Diferentes versiones de este Manual (BUENOS AIRES, 2008; 2009; 2011; 2013) evidencian que Fortalecimiento aspiraba a producir nuevos desempeños para docentes y estudiantes. En cuanto a los docentes se propuso integrarlos en equipos de trabajo que desarrollaran actividades dentro y fuera del aula no limitadas a sus experticias técnicas o profesionales, modificando así los modos habituales de trabajo reducidos a la enseñanza de asignaturas $o$ áreas de conocimiento específicas dentro del aula. Los estudiantes son explícitamente considerados actores escolares con capacidad para incorporar la relevancia y la necesidad de completar el nivel medio para sus vidas sociales y ocupacionales. En cuanto a los proyectos, el PFIEM reconoce distintos tipos de iniciativas. En el año 2013, por ejemplo, se destacaron los Proyectos que revisan la enseñanza y la propuesta formativa de la escuela, los Proyectos de apoyo, seguimiento $y$ orientación de las trayectorias escolares, y los Proyectos y Programas dirigidos a la Escuela Secundaria, tales como pasantías en el programa Aprender Trabajando.

Desde una perspectiva de política instrumental que considera a la política como una herramienta racional y técnica utilizada por los responsables políticos (como las autoridades educativas, asesores pedagógicos, asesores técnicos), Fortalecimiento podría entenderse como una estrategia desplegada para resolver problemas y promover cambios en las escuelas estatales. Sin embargo, algunos de los cambios experimentados como importantes para los propios docentes que produjo la implementación de proyectos, afectaron facetas de su trabajo y relaciones en la escuela y provocaron cambios no referidos en los documentos oficiales.
Para mostrar esto tomaremos como ejemplo la asignación de módulos institucionales (en adelante MI) para pagar el trabajo de profesores en los proyectos de las escuelas.

La asignación de fondos para esos módulos se distribuye por proyecto y por escuela según una fórmula que tiene en cuenta determinados componentes: cantidad de matrícula; total de alumnos/as repetidores, que abandonaron (salidos sin pase); promovidos al siguiente año escolar; con sobre-edad; cantidad de turnos y uso efectivo de los módulos asignados el año anterior (BUENOS AIRES, 2008). No hemos encontrado documentos oficiales que indiquen cuál es el peso relativo o la operación aritmética entre los componentes de la fórmula. Los docentes y autoridades escolares tampoco conocen cómo se realiza el cálculo de esa fórmula. De ahí que no es posible estimar la cantidad de MI que cada escuela debe recibir por los proyectos cada año. Este manejo del presupuesto es considerado por docentes y supervisores como poco transparente, sobre todo porque en la guía oficial utilizada para solicitar MI explícitamente se enuncia que el monto que se asigna no puede ser cuestionado por las escuelas, debido a que lo que reciba una escuela no puede ser alterado sin afectar el monto asignado a otras (BUENOS AIRES, 2008). Ahora bien, los MI se materializan en dinero que se paga mensualmente. Cada MI es equivalente a cuarenta minutos de trabajo, o sea al tiempo de duración de una clase y los docentes cobran por MI asignados según su integración a proyectos. Queda introducida así, una nueva forma de retribución por el trabajo docente que se superpone a la instituida -los salarios de acuerdo con escalas predefinidas establecidas por el Estatuto del Docente, normativa que regula aspectos centrales de la contratación, condiciones laborales y carrera de los docentes.

A esta situación se agrega que el/la director/a es la persona responsable en cada escuela de decidir quiénes reciben MI y cuántos les corresponden. Esto implica tomar decisiones 
sobre el valor que se asigna a cada proyecto y sobre el trabajo de los docentes desempeñado en cada caso. Si tomamos en cuenta la relevancia de las relaciones personales en la designación de autoridades escolares en el caso de las EMEM y en la constitución de una parte del plantel docente, podremos advertir que una estrategia poco transparente a nivel de asignación de recursos por parte de la agencia estatal promueve un aumento del poder discrecional de las autoridades de las escuelas y expresa e implica formas inéditas de regulación y control a nivel local por parte de los directores de escuela.

Desde sus inicios, Fortalecimiento ha exigido que los directores de escuela fueran los responsables de la selección de los proyectos y de los docentes a los que se les asignaba los MI. Asimismo, son los/as responsables de elevar la solicitud de fondos a la supervisión y al PFIEM. Los supervisores deben aprobar los proyectos que hasta principios de 2014 eran evaluados por el Comité de Coordinación de Fortalecimiento. De acuerdo con documentos oficiales, los directores también son responsables de controlar el cumplimiento de los proyectos y del trabajo y asistencia de los docentes involucrados (BUENOS AIRES, 2008; 2009; 2011). La introducción de los MI ha implicado la creación de un circuito oficial paralelo de asignación de recursos a los docentes. Si bien los directores no pueden seleccionar formalmente a los docentes para sus escuelas (aunque en muchos casos lo hacen), deben elegirlos para la realización de los proyectos de Fortalecimiento y de otro programa denominado Plan de Mejora. Los términos de contratación de los docentes, de manera similar a la etapa de creación de la escuela de nuestro estudio, no siguen los lineamientos establecidos por el Estatuto del Docente que exigen la presentación de antecedentes ante una Junta de Clasificación Docente que jerarquiza a los candidatos según el puntaje total que hayan obtenido para luego otorgar los cargos vacantes en las distintas escuelas. La nueva modalidad de incorporación de personal da cuenta de una redefinición en términos de favores, influencias, cercanías, contraprestaciones entre directores $\mathrm{y}$ docentes que introduce como legítima una práctica informal, ajena a la normativa existente, pero consonante con reglas relativamente aceptadas entre quienes trabajan en las escuelas. Vemos así que la directora ya no se comporta como una colega con funciones de autoridad superior en aspectos pedagógicos, didácticos, administrativos, burocráticos, sino que cumple funciones de empleadora o gerente y el/la docente pasa a virtualmente a ser empleado/a de esta directora. Ambos pierden ciertas prerrogativas y conquistan otras que, en cualquier caso, precarizan el trabajo docente, así como tensionan y desacomodan la organización, el funcionamiento y las relaciones entre sujetos y grupos en la escuela. El gobierno, como operador del Estado, al redefinir la modalidad de contratación de docentes y trabajadores en las escuelas también redefinió en un aspecto relevante la modalidad de intervención y control en la escuela. Produjo así un alejamiento del esquema de relaciones entre Estado y escuelas que rigió con anterioridad a la década del 90 y un desplazamiento hacia una regulación informal, mediante contactos informales y gestiones personales que tiende más a desarticular y desorganizar una institución, que a producir un funcionamiento autónomo, fomentar la participación, y redistribuir el poder.

\section{Formas de regulación local y condiciones de trabajo docente: el caso de El Puente}

El Puente fue creado en el $2006 \mathrm{y}$, desde entonces, ha sido financiado por el Programa de Fortalecimiento. El Puente se inscribe en una línea prioritaria del PFIEM: Proyectos de apoyo, seguimiento y orientación de las trayectorias escolares. En el lenguaje del PFIEM, este proyecto brinda apoyo a la terminalidad de los estudios secundarios, para la preparación de materias previas y evaluaciones parciales y para el apoyo a la cursada de asignaturas consideradas críticas. 
En los años 2013 y 2014, este proyecto contaba con la mayor cantidad de MI. Esta situación reflejaba el reconocimiento de las autoridades hacia su coordinadora, Bety, y que -en palabras de la directora de la escuela- este era un proyecto "con el que podemos lucirnos" y que "tenía resultados concretos" (Nota de campo, 5 de diciembre 2013). En un contexto de mayores presiones por incluir, El Puente adquirió centralidad e importancia porque aumentaba el número de graduados de la escuela. Así, este proyecto lograba que muchos estudiantes del último año de estudio se graduaran luego de haber egresado sin terminar su último año de estudio.

La centralidad de este proyecto es relativamente reciente. Su ritmo de crecimiento sólo puede entenderse en el marco de la historia de la escuela y las maneras específicas que asumieron las reglas del juego que se fueron configurando en la escuela para definir quiénes obtuvieron recursos del PFIEM en distintos momentos. El Puente fue creado durante el período que la escuela fue dirigida por la segunda directora. La primera directora había rechazado la idea de crear este proyecto debido a la existencia de conflictos con el director de la escuela primaria. Bety (creadora de El Puente) nos habló de lo que la impulsó a poner en marcha este proyecto:

Otra que siempre tuve en la mira es que tuvieran un lugar amable. ¿Viste como cuando un chico vuelve a la casa y hace las tareas? ¿y tiene la leche o la merienda? ésa era mi idea. Que se sintiera bien, (...) que uno le pudiera decir: mirá, a ver si ves esta oración, a ver qué te parece, dar más herramientas que, por a veces falta de tiempo, en el aula no se puede (...) si creamos mejores condiciones, todos tienen condiciones para aprender (...). $E l$ Puente tenía que ofrecer eso. (Entrevista con Bety, 9 de noviembre de 2013).

Bety quería abrir la biblioteca para que los estudiantes tuvieran un lugar para "leer", “acceder a la información”, y para "hablar de lo que ellos quisieran” (Entevista con Bety, 9 de noviembre de 2013), antes de la jornada escolar ${ }^{12}$. Según ésta y otros docentes, los estudiantes no tenían un lugar físico propio en sus hogares. Muchos de ellos residian con varios adultos y hermanos en pequeñas casas precarias y no tenían espacio físico para estudiar o para guardar materiales para leer, estudiar o hacer los deberes.

Bety nos contó que a principios de los 2000 no tenía información sobre los proyectos ni sobre los criterios que la dirección utilizaba para seleccionarlos y ponerlos en marcha. Recién se enteró de la existencia del Programa Fortalecimiento a través de la segunda directora, Martina, quien la puso al tanto de que podía solicitar MI. En palabras de Bety:

A mí la que me avivó fue Martina ¿Cómo que hay proyectos y yo nunca los vi? pero ¿dónde están? ¿quién los dirige?, pero ¿cuándo lo hacen? (...) No sabía cómo hacían esos docentes. Yo pensaba que la dirección le decía a tales personas por su idoneidad que los hicieran. Hasta que Martina me dijo: no, el que quiere escribe un proyecto y lo tiene que entregar. Yo no sabía cómo escribir un proyecto. Ella me invitó a su casa. Hablábamos y ella escribía el proyecto. Si no hubiera sido por ella yo no me hubiese presentado. (Entrevista con Bety, 9 de noviembre de 2013).

Esta falta de publicidad de la existencia de fondos para proyectos, de la forma de solicitarlos, y de los criterios utilizados por la dirección persistía durante nuestro trabajo de campo. Varios profesores nos contaron que no sabían de dónde venían los fondos de los proyectos (si eran de Fortalecimiento o del Plan de Mejora o de la propia escuela). Tampoco conocían cómo eran elegidos los docentes que llevaban adelante estas actividades, ni cómo se decidía la cantidad de recursos económicos que se les asignaría. Por ejemplo, Marisa, profesora

12- Antes de El Puente, la biblioteca de la escuela secundaria sólo estaba abierta durante el horario escolar y raramente era utilizada por los estudiantes. 
de plástica, nos contó cómo la directora no la tuvo en cuenta para hacer un mural con los chicos en uno de los pasillos de la escuela con dinero de un proyecto. Ella no entendía por qué habían elegido "al amigo del asistente pedagógico que no trabajaba en la escuela para hacer ese trabajo" (Nota de campo, 21 de marzo de 2014). Marisa era una docente que tenía formación en artes plásticas, dictaba varias materias en la escuela y era muy valorada por Bety y otros docentes. Bety también compartió con nosotras esa sensación de injusticia y también bronca porque los módulos habían sido asignados a un amigo del asesor pedagógico, "que no tiene nada que ver en la escuela" (Nota de campo, 21 de marzo de 2014).

En el caso del proyecto El Puente, la gran mayoría de los docentes con los que conversamos conocían su existencia, pero no todos sabían cómo era financiado, ni tampoco cómo se había seleccionado a los docentes que lo integraban ni a su coordinador. La escasa visibilidad de los proyectos así como de los criterios para seleccionarlos también se hizo evidente en la última Jornada Institucional en 2013. El objetivo de esa reunión era informar a docentes y preceptores sobre los proyectos, sus objetivos y sus relaciones con el Proyecto Escuela (PE). Esa fue la primera vez que las autoridades escolares realizaron una reunión con el personal docente para conversar sobre los proyectos y la necesidad de articularlos en el Proyecto Escuela (PE). Las presiones de las autoridades educativas nacionales y locales, a través de las supervisiones ampliadas que se empezaron a realizar en el 2013, habían influido en la definición de la agenda de esa Jornada Institucional. Cuando el profesor que coordinaba la reunión preguntó a los colegas si sabían cuáles eran los proyectos que existían en la escuela, la mayoría manifestó desconocimiento. En varias ocasiones, fuera de ámbitos formales de reunión, varios profesores nos habían comentado que nunca habían visto el PE, que no sabían quién hacía los proyectos y para qué, tampoco cómo ni quiénes lo financiaban.
Durante nuestro trabajo de campo observamos cómo la directora y la vicedirectora jugaban un rol central en la selección de proyectos y en el uso de los recursos humanos y económicos para los mismos. En esta selección ingresaban como un elemento muy relevante las relaciones personales que ellas tenían con distintos docentes y grupos de docentes de la escuela. Ellas explícitamente nos expresaron que elegían a los docentes de acuerdo a pautas que nombraban como la confianza, saber que van a trabajar, y que se comprometen con la escuela. Esta confianza y reconocimiento del compromiso del otro se anclaban en relaciones personales -ya sea de amistad, parentesco o basada en afinidades de distinto tipo- que podían haberse forjado fuera de la escuela o luego de haber ingresado en la escuela y a partir de caer muy bien a alguna de las personas cercanas a la dirección. Así, por ejemplo, a pedido de Bety antes de jubilarse, la directora nombró a Faustino como coordinador de El Puente. Para Bety, él era su sucesor e iba a ser capaz de continuar y mejorar el trabajo que se estaba haciendo en El Puente. La palabra de Bety era muy valorada por la directora, quien también expresó en numerosas oportunidades el buen trabajo que hacía Faustino tanto en El Puente como en sus clases. La directora también decidió asignar módulos institucionales de El Puente a Juan para realizar tareas que no tenían que ver con este proyecto. Esta situación era conocida por los docentes de El Puente. La directora le asignó MI para que arreglara las netbooks del programa Conectar Igualdad, tarea que no recibía ningún financiamiento específico y, según Juan y otros docentes, se había convertido en un gran problema. Juan nos contó que tenía que trabajar más horas que las de los módulos para intentar cumplir con esa tarea.

\section{Discusión}

Las EMEM nacieron como escuelas con niveles de autonomía mayores que las escuelas secundarias nacionales que co-existían en la ciudad de Buenos Aires y que pocos años 
después de haber sido creadas las primeras, fueron municipalizadas. En estos primeros años, la directora fundadora de la escuela estudiada eligió a muchos docentes y preceptores, forjó relaciones estrechas con familias y estudiantes, con organizaciones no gubernamentales, universidades. Como sintetizó una docente intentando contar quién había sido Susana allí: "ella era como una madre [que] todo lo contenía [y que] tenía que estar en todo" (Nota de campo, 15 de mayo de 2014). Esa forma de liderazgo de esta directora no volvió a repetirse, la escuela se fue institucionalizando cada vez más y quienes la sucedieron ejercieron una forma de dirección escolar más convencional. Sin embargo, como hemos mostrado, las directoras continuaron teniendo márgenes de maniobra para disponer y distribuir recursos monetarios entre docentes (aunque con variables volúmenes en distintos momentos, llegando a un máximo en el año 2013 por el funcionamiento de Fortalecimiento y Mejoras) y para seleccionar a algunos docentes por vías informales.

Hemos mostrado cómo operaba en la EMEM una forma de regulación local, situada y activa que da cuenta de cómo los actores escolares se re-apropiaban y re-definían las lógicas que orientaban la distribución de recursos materiales y simbólicos. Esta lógica podría pensarse como regulación autónoma, expresión que utilizan Dupriez y Maroy (2003) para dar visibilidad a las maneras en que los actores sociales juegan y participan en la producción de orientaciones a la acción. La comprensión de lo que hacen en términos de juegos, apunta a reconocer las capacidades de los actores sociales para producir y poner en acción o rechazar, reglas instaladas como parte de la regulación institucional del estado.

El análisis precedente también ha mostrado como el PFIEM reforzó el rol de la directora de la escuela y amplió sus capacidades para decidir qué tipo de proyectos y a qué docentes se financiaba, instalando maneras informales -y a veces poco trasparentes- para la distribución de recursos, reguladas por las relaciones de confianza y afinidad (ya sea personal, fundada en el parentesco, o en la militancia social con poblaciones similares a las que concurre a la escuela). Este reforzamiento del rol de la directora era acompañado por la intensificación de su propio trabajo. La directora tenía que conversar con los docentes sobre proyectos en marcha o nuevos; decidir cuáles continuaban y cuántos módulos deberían tener; completar variedad de formularios junto con el asesor pedagógico; armar el documento PE; y tener reuniones con la asesora de Fortalecimiento y con el supervisor. Estas tareas se sumaban a las muchas que el equipo de conducción tenía que llevar adelante cotidianamente. En el año 2014, debido a las nuevas presiones de las autoridades educativas locales, la dirección también tenía que convocar y organizar las reuniones con el Consejo Consultivo para definir el PE y decidir los proyectos para los cuales se solicitaría financiamiento. También a partir de ese año, el equipo de conducción debía participar de supervisiones ampliadas a las que debían asistir el supervisor de la escuela y las Asistentes Técnicas del PM y del PFIEM, con el objetivo de acompañar a la escuela en la elaboración del PE y en la articulación de éste con los proyectos. Asimismo, la directora debía hacerse cargo del manejo de dinero del Plan de Mejoras -el cual también otorgaba módulos institucionales (MI)-. Por esta razón tenía que buscar fondos en una cuenta de ahorro en su nombre, fuera del horario escolar y asumiendo riesgos personales por la manipulación de dinero. Sorprendentemente, esta sobrecarga de trabajo no era cuestionada -tal vez podíamos escuchar alguna queja. Ella aceptaba esta modalidad y argumentaba que lo hacía porque así se conseguían más cosas para los chicos.

Asimismo, la introducción de MI por parte del PFIEM evidencia nuevas formas de regulación institucional del trabajo de las docentes que participan en estos proyectos escolares. Para los docentes, los MI eran parte del salario, porque se abonaban junto con los sueldos mensuales y se volcaban a los recibos 
de sueldo como sumas no remunerativas, lo cual implica que esas sumas no incluyen obligaciones impositivas ni porcentuales de beneficios, no están sujetas a descuentos y no se la tiene en cuenta para ningún fin. En los últimos años, especialmente en el sector público, los trabajadores perciben sumas de dinero con carácter no remunerativo. El Estado, frente a los reclamos salariales, aumenta el salario de bolsillo de sus empleados utilizando el carácter no remunerativo y así evita pagar, por ejemplo, las cargas sociales. Los sindicatos docentes han denunciado las sumas no remunerativas dentro del salario como dinero en negro. Los MI como modalidad de suma no remunerativa dentro del salario, pueden explicarse como continuidad de los procesos de precarización laboral desplegados sobre el trabajo docente en la década de 1990 (SÁNCHEZ CERÓN; DEL SAGRARIO CORTE CRUZ, 2012). Algunos docentes se han referido a los MI como ahorro forzoso porque, por lo general, el dinero que cobraban lo recibían todo junto al final del año escolar.

La inseguridad, la incertidumbre y la falta de garantía de las condiciones socio-económicas, reflejadas en cuándo se van a empezar a pagar los módulos, cuántos meses van a ser efectivamente pagados, quiénes van a recibir ese pago, hasta cuándo lo van a cobrar, etc., formaba parte del trabajo cotidiano de los docentes y frecuentemente esos rasgos estaban sostenidos por discursos, muchas veces de los propios docentes, que no reconocían este proceso de profundización de la precarización. En la EMEM de nuestro estudio, los docentes también solían utilizar dinero propio para comprar materiales didácticos, trasladar equipos, etc. y trabajaban más horas de las que se consideran en los MI, etc. Éstas eran las condiciones que los docentes consideraban normales -con mayor o menor grado de incomodidad o resignación. Esta nueva normalidad era el resultado de una manera de gobernar las escuelas promovida por el Estado.

Esta lógica no puede ser entendida sólo como el resultado de una crisis o situación irregular, o como una forma de superar los problemas que dejó el neoliberalismo de los 90 y mucho menos, como parte de los beneficios de la expansión de la escuela media y la inclusión de más jóvenes. Antes bien, debe ser comprendida a partir de los efectos que produce en las prácticas de las vidas cotidianas de los sujetos empujados a trabajar en las condiciones y con esas relaciones y, también en los discursos que constituyen esas vidas cotidianas, por su capacidad performativa y subjetivadora. La introducción del PFIEM ha ido instalando paulatinamente ciertos lenguajes y vocabularios - proyecto, objetivos, estrategias, indicadores, evaluación- y ciertas maneras legítimas de presentar y registrar tanto lo que hace la escuela, que debe quedar inscripto en el PE, como lo que hacen los docentes en los proyectos. Los docentes coordinadores de cada proyecto tenían que presentarlos al equipo de conducción siguiendo lineamientos generales, que se han estandarizando año a año en torno a cada vez más dimensiones o categorías previamente establecidas por el PFIEM. Así, escribir proyectos y mostrar el trabajo de la escuela a partir del PE y sus resultados es una nueva actividad que supone que autoridades y docentes sean capaces de: i) reconocer la existencia de este tipo de financiamiento así como las reglas de juego que suponen su obtención y utilización; ii) movilizarse (individual o grupalmente) para obtener el apoyo de los niveles superiores (ya sea del supervisor en el caso de la directora o de la directora en el caso de los docentes); y iii) inscribir textualmente lo que se hace y quiere hacer, utilizando ciertas palabras y expresiones legítimas y autorizadas por Fortalecimiento.

Los PEs muestran que la inclusión de los proyectos se ha vuelto una estrategia central para dejar registrado lo que pasa en la escuela y cómo se utilizan recursos humanos y materiales para desarrollar actividades separadas del plan de estudios, que apuntan a transformar maneras de enseñar y aprender. Sin embargo, ser docente no garantiza acceder a la información sobre programas, ni oportunidades para presentar proyectos, ya que como mostramos más arriba, 
muchos profesores ni siquiera saben qué proyectos tiene la escuela en la que trabaja, ni cómo escribirlos y tampoco cuentan con la modalidad de relación que requiere esta otra tarea. A pesar de ello, están afectados por un clima de incertidumbre, de velada competencia por recursos materiales y simbólicos, y de diferenciación entre grupos de docentes según su grado de proximidad o distancia respecto de la dirección escolar.

\section{Consideraciones finales}

En este trabajo hemos mostrado cómo el análisis centrado en lo que hacen los actores en instituciones y situaciones concretas con las políticas educativas permite acceder a los efectos reales de esas políticas sobre las instituciones, los actores y sus estrategias frente a los cambios inducidos desde arriba y, por lo tanto, al sentido último de esas políticas, más allá de los propósitos enunciados por las mismas. El enfoque etnográfico adoptado iluminó la reconfiguración de nuevas formas de control y regulación del Estado, tal como se está dando a través de una lógica práctica, más allá de los discursos estatales. Tomando en consideración este proceso, emergen los datos de una continuidad, que demuestra una vez más que los procesos políticos, sociales y culturales no siguen puntualmente los cortes de los períodos presidenciales, ni los cambios se corresponden a los que el Estado suele decir de sí mismo para trazar la línea de separación con el pasado que todavía gravita negativamente $\mathrm{y}$ se reproduce en varios aspectos en el presente.

\section{Referencias}

BALL, Stephen; MAGUIRE, Meg; BRAUN, Annette. How schools do policy, Policy enactments in secondary schools. Abingdon: Routledge, 2012.

BALL, Stephen; YOUDELL, Deborah. Hidden privatisation in public education: preliminary report educational international - 5th World Congress, Brussels: Education International, 2007.

BARROSO, João. Organização e regulação dos ensinos básico e secundário, sentidos de uma evolução. Educação \& Sociedade, Campinas, v. 24, n. 82, p. 63-92, 2003.

BUENOS AIRES. Ministerio de Educación. Pautas para la elaboración de proyectos y la utilización de módulos institucionales para los establecimientos de nivel medio dependientes de la DEM, la DET, la DEA y la DFA. Buenos Aires: Ministerio de Educación. Gobierno de la Ciudad de Buenos Aires, 2009.

BUENOS AIRES. Ministerio de Educación. Pautas para la elaboración de proyectos y la utilización de módulos institucionales para los establecimientos de nivel medio dependientes de la DEM, la DET, la DEA y la DFA. Buenos Aires: Ministerio de Educación. Gobierno de la Ciudad de Buenos Aires, 2011.

BUENOS AIRES. Ministerio de Educación. Programa Fortalecimiento Institucional de la Escuela Media Encuadre y algunas líneas de trabajo - marzo (a modo de anticipo del Manual Pautas para la elaboración de proyectos y la utilización de Módulos Institucionales /2013). Buenos Aires: Ministerio de Educación. Gobierno de la Ciudad de Buenos Aires, 2013.

BUENOS AIRES. Ministerio de Educación. Resolución n 8.648 - MEGC/08. Buenos Aires: Ministerio de Educación. Gobierno de la Ciudad de Buenos Aires, 2008.

CARNOY, Martín; MOURA CASTRO, Claudio. ¿Qué rumbo debe tomar el mejoramiento de la educación en América Latina? Banco Interamericano de Desarrollo, 1996. Disponible en: <http://services.iadb.org/wmsfiles/products/Publications/364089. pdf>. Acceso en: 22 feb. 2016.

DABENIGNO, Valeria et al. Iniciativas institucionales para la retención y promoción de los aprendizajes en el primer año de la escuela secundaria. Buenos Aires: Ministerio de Educación, Gobierno de la Ciudad de Buenos Aires, 2014.

DUPRIEZ, Vincent; MAROY, Christian. Regulation in school systems: a theoretical analysis of the structural framework of the school system in French-speaking. Journal of Education Policy, v. 18, n. 4, p. 375-392, 2003. doi.org/10.1080/0268093032000106839 
DURO, Elena; PERAZZA, Roxana (Org.). Acerca de la obligatoriedad en la escuela secundaria argentina. Análisis de la política nacional. Buenos Aires: Unicef, s/f.

FELDFEBER, Myriam. La redefinición del espacio público: el caso de las escuelas autogestionadas en Argentina. Educação \& Sociedade, Campinas, v. 24, n. 84, p. 923-943, 2003.

FELDFEBER, Myriam; GLUZ, Nora. Las políticas educativas en Argentina. Herencias de los '90, contradicciones y tendencias de "nuevo signo". Educação \& Sociedade, Campinas, v. 32, n. 115, p. 339-356, 2011.

GIOVINE, Renata. El arte de gobernar el sistema educativo: discursos de estado y redes de integración socioeducativas. Quilmes: Universidad Nacional de Quilmes, 2012.

GOROSTIAGA, Jorge. La democratización de la gestión escolar en la Argentina: una comparación de políticas provinciales. Archivos Aanalíticos de Políticas Educativas, v. 15, n. 2, 2007. Disponible en: <http://epaa.asu.edu/ojs/article/viewFile/50/176>. Acceso en: 11 mar. 2016.

MAROY, Christian. Regulations and inequalities in European Education Systems: final report. Bruselas: Université Catholique de Louvain Girse, 2004.

MAROY, Christian; DUPRIEZ, Vincent. La régulation dans les systémes scolaires: proposition theórique et analyse du cadre structurel en Belgique francophone. Revue Française de Pédagogie, Lyon, n. 130, p. 73-87, 2000.

MÁS ROCHA, Stella Maris. Un proyecto de educación secundaria en los '90. El caso de las Escuelas Municipales de Educación Media (EMEM) de la ciudad de Buenos Aires. Buenos Aires: Universidad Nacional de Luján, 2006. Informe final (Beca de Investigación, Categoría de Iniciación). 75 p.

MONTESINOS, María Paula; SCHOO, Susana. La implementación de los Planes de Mejora Institucional: aportes para las políticas orientadas al fortalecimiento de la educación secundaria obligatoria. Buenos Aires: Dirección Nacional de Información y Evaluación de la Calidad Educativa (DiNIECE), 2014.

REYNAUD, Jean-Daniel. Les rèfles du jeu: I'action collective et la régulation social. Paris: Armand Colin, 1997.

SÁNCHEZ CERÓN, Manuel; DEL SAGRARIO CORTE CRUZ, Francisca María. La precarización del trabajo: el caso de los maestros de educación básica en América Latina. Revista Latinoamericana de Estudios Educativos, México D.F., v. 42, n. 1, p. 25-54, ene./mar. 2012.

SINISI, Liliana et al. El programa de fortalecimiento institucional en las escuelas medias: un estudio sobre los procesos de apropiación, negociación y resistencias en la relación política educativa y cotidianidad escolar. Buenos Aires: Secretaría de Educación del Gobierno de la Ciudad de Buenos Aires, 2003.

TEDESCO, Juan Carlos; TENTI FANFANI, Emilio. La reforma educativa en la Argentina: semejanzas y particularidades. Las Reformas Educativas en la década de 1990. Un estudio Comparado de Argentina, Chile y Uruguay. Buenos Aires: BID/Ministerios de Educación de Argentina, Chile y Uruguay; Grupo Asesor de la Universidad de Stanford, 2004.

TENTI FANFANI, Emilio; FREDERIC, Sabina; STEINBERG, Cora. Abandono escolar y políticas de inclusión en la educación secundaria. Buenos Aires: IIPE Unesco - PNUD, 2009.

WHITTY, Geoff; POWER, Sally. Marketization and privatization in mass education systems. International Journal of Educational Development, v. 20, n. 2, p. 93-107, 2000. Disponible en: <https://www.journals.elsevier.com/international-journal-ofeducational-development/>. Acceso en: 20 mar. 2016.

Recibido en: 23.02.2016

Aprobado en: 17.05.2016

Analía Inés Meo es socióloga (Universidad de Buenos Aires) y doctora en Sociología (Universidad de Warwick). Investigadora del CONICET y profesora de la carrera de Sociología de Facultad de Ciencias Sociales (UBA) en Argentina. Se ha especializado en el área de sociología de la educación.

Diana Milstein es doctora en Antropología Social. Profesora de la Facultad de Educación de la Universidad Nacional del Comahue e investigadora del Centro de Investigaciones Sociales (CONICET-IDES), Argentina. Se ha especializado en antropología de la educación y etnografía con niñ@s, antropología del cuerpo y etnografía. 